\title{
Lactulose, but not Macrogol or Bisacodyl, Shows a Prebiotic Effect in a Computer-Controlled In Vitro Model of the Human Large Intestine
}

\author{
Melanie K. Bothe ${ }^{1 *}$, Annet J.H. Maathuis ${ }^{2}$, Susann Bellmann ${ }^{2}$, Katja Lange ${ }^{2}$, Jos M.B.M. van der Vossen ${ }^{3}$, Annalena Neumann ${ }^{1}$, \\ Angelika Kuchinka-Koch ${ }^{4}$ and John F. Stover ${ }^{1}$ \\ ${ }^{1}$ Fresenius Kabi Deutschland GmbH, Else-Kroener-Strasse 1, 61352 Bad Homburg, Germany \\ ${ }^{2}$ TIM B.V., formerly part of Triskelion, P.O. Box 343, 3700 AV Zeist, The Netherlands \\ ${ }^{3}$ The Netherlands Organisation for Applied Scientific Research (TNO), Microbiology and Systems Biology Department, P.O. Box 360, 3700 AJ Zeist, \\ The Netherlands \\ ${ }^{4}$ Fresenius Kabi Austria GmbH, Estermannstrasse 17, 4020 Linz, Austria
}

${ }^{*}$ Corresponding author: Melanie K. Bothe, Fresenius Kabi Deutschland GmbH, Else-Kroener-Strasse 1, 61352 Bad Homburg, Germany; Tel: +49 6172 686 6768; E-mail: melanie.bothe@fresenius-kabi.com

Received: November 09, 2021; Accepted: November 15, 2021; Published: November 17, 2021

\begin{abstract}
Background: Patients with chronic constipation often suffer from dysbiosis and may benefit from prebiotic effects of laxatives.

Methods: Here we evaluate potential beneficial effects on the gut microbiome of the most commonly used laxatives Macrogol, Bisacodyl, and Lactulose in their usual daily dose for adults using the TIM-2 system, a computer-controlled model of the proximal large intestine with metabolically active, anaerobic microbiota of human origin.
\end{abstract}

Results: Only Lactulose increased the short-chain fatty acid levels and decreased the branched-chain fatty acid levels, $\mathrm{pH}$, and ammonia. Five days of incubation with Lactulose increased the bacterial counts of Bifidobacterium and Lactobacillus which was not observed with Macrogol or Bisacodyl.

Conclusion: These data show that Lactulose, in contrast to Macrogol and Bisacodyl, exerts a prebiotic effect when compared in the same in vitro system.

Keywords: Lactulose, Microbial fermentation, Bifidobacteria, Lactobacilli, Laxative

\section{Introduction}

Dysbiosis in patients with constipation is not yet fully understood, but consists of increased counts of mucosal Bacteroides species and decreased fecal bifidobacteria and lactobacilli $[1,2]$. The reduced abundance of beneficial bacteria in constipated patients may be ameliorated by prebiotic laxatives. According to guidelines, Macrogol, Bisacodyl or its derivative sodium picosulfate are agents for first-line therapy of constipation [3], while Lactulose is frequently recommended for chronic constipation by pharmacies [4]. During pregnancy, Macrogol and Lactulose are recommended as first-line therapy [5] which is in line with general practice [6], whereas during lactation, Macrogol, Lactulose, Bisacodyl or sodium picosulfate may be used [5].

To date, Lactulose is clearly considered a prebiotic laxative [7-10]. However, only limited data are available regarding the prebiotic effects of Bisacodyl and Macrogol. Macrogol consists of polyethylene glycol (PEG). In general, PEG is known to affect the intestinal microbiota. Phylotype richness was reduced in PEG-induced diarrhea in human, while phylotype diversity and evenness were unaffected [11]. In rats
[12], PEG treatment increased the number of Verrucomicrobia and decreased that of Firmicutes. In mice, Macrogol 3350/PEG decreased the microbial density [13] and relative abundance [14], while Lactulose increased it [13]. Bisacodyl increased the gut microbiota metabolites namely SCFA in rats [15]. Furthermore, a slight increase in bifidobacteria was observed after three months of constipation treatment in humans [1].

Data on direct comparison of the prebiotic effects of the three laxatives Lactulose, Macrogol and Bisacodyl are sparse. In patients with constipation, the efficacy of Lactulose was similar to that of PEG in relieving constipation in a 4 week treatment [16]. The levels of bifidobacteria, but not lactobacilli, were significantly increased in the patients receiving Lactulose, but not in the patients receiving PEG [16]. In contrary, the total amount of bacteria was rather decreased and the colonic fermentation inhibited by treatment with PEG [16]. To our knowledge, no further studies directly comparing at least two of the three laxatives are available, hindering the comparison of Lactulose, Bisacodyl and Macrogol regarding their prebiotic effect. 
In our study, we investigated the prebiotic effect of Lactulose, Macrogol, and Bisacodyl in the TIM-2 model, an in vitro model of the proximal colon. The results of this study demonstrate that Lactulose contrary to Macrogol or Bisacodyl, increased the short-chain fatty acid production as well as the bifidobacterial and lactobacilli count, thereby showing a prebiotic effect.

\section{Materials and Methods}

\section{Informed Consent}

This is an in vitro study. It does not require IRB approval or informed consent.

\section{Test Product}

In this study Laevolac (Fresenius Kabi Austria GmbH, Linz, Austria), an oral solution containing $670 \mathrm{mg} / \mathrm{mL}$ Lactulose, Macrogol 3350 (Norgine B.V., Amsterdam, The Netherlands) and Bisacodyl (Boehringer Ingelheim, Ingelheim, Germany) were used. Experiments without test products served as negative control.

\section{Intestinal Conditions of the TIM-2 System}

The TNO intestinal model TIM-2 is a dynamic in vitro model of the proximal colon $[17,18]$. In this system, essential parameters were maintained at standardized conditions: body temperature; $\mathrm{pH}$ in the lumen of the proximal colon ( $\mathrm{pH} 5.8$ ); delivery of a pre-digested substrate from the 'ileum' (SIEM); mixing and transport of the intestinal contents; dialysis-driven absorption of water and metabolic products. In addition, the system was strictly maintained anaerobic by flushing with nitrogen. Fermentation products, metabolites and other low molecular weight compounds were steadily removed from the lumen via dialysis using a semipermeable membrane system within the colon compartment.

SIEM (standardized ileum efflux medium) simulates the material passing the ileocecal valve in humans reaching the colon. SIEM was prepared as described previously [18-20] and contains the major nondigestible carbohydrates (pectin, xylan, arabinogalactan, amylopectin, starch) found in a normal western diet as well as protein (bactopepton, casein), some ox-bile, Tween 80 , vitamins, and minerals. SIEM was added to the system at a speed of $2.5 \mathrm{ml} / \mathrm{h}$. The speed of dialysis was $1.5 \mathrm{ml} / \mathrm{min}$.

During the experiment, the intestinal contents were mixed continuously by the peristaltic movements of the TIM-2 system. The $\mathrm{pH}$ was maintained at $\mathrm{pH} 5.8$ or above by automatic titration (minute by minute) with $2 \mathrm{M} \mathrm{NaOH}$. The amount of administered $\mathrm{NaOH}$ was monitored, allowing to draw conclusions about the acid production induced by the different test compounds.

Before each experiment the secretion fluids and dialysis solutions were freshly prepared, the $\mathrm{pH}$ electrodes calibrated, and new membrane units were installed. The system was inoculated with a standardized microbiota of human origin, one day before the start of the test period. This standardized microbiota was prepared using fecal donations from a group of 4 healthy volunteers (1 male, 3 females (non-pregnant, non-lactating), age $38.8 \pm 3.9$ years; BMI $24.2 \pm 1.5$ $\mathrm{kg} / \mathrm{m} 2$ ) as described [21]. After overnight adaptation the $120 \mathrm{~h}$ test period started.

\section{Addition of the Test Product}

The test products were added to the system at their indicated daily doses for adults, i.e. $10 \mathrm{~g} /$ day Lactulose, $13.125 \mathrm{~g}$ /day Macrogol 3350, or $5 \mathrm{mg} /$ day Bisacodyl. Test products Lactulose and Macrogol were mixed 'as is' through the SIEM (described in more detail below) and added (semi-)continuously during the entire test period. Before its administration to TIM-2, Bisacodyl was incubated for $3 \mathrm{~h}$ in TIM2 dialysate at $\mathrm{pH} 7.2$ and subsequently overnight at $\mathrm{pH}$ 5.8. This measure allowed to soften the outer enteric coating of the formulation and to release Bisacodyl appropriately in TIM-2. Both the dialysate and the formulation were added as a daily bolus. The control runs were performed in quadruplicate, while the test products were studied in triplicates (Lactulose) or duplicates (Macrogol, Bisacodyl).

\section{Sampling from TIM-2}

Metabolites including the short-chain fatty acids (SCFA), branched-chain fatty acids (BCFA), ammonia and lactate produced in TIM-2 were continuously separated from the lumen using a semipermeable membrane unit. Dialysates were collected at the start of the test period and after $24,48,72,96$, and $120 \mathrm{~h}$, respectively. Volumes were measured and samples were taken from the dialysates.

Luminal samples taken at the beginning and end of the experiment $(\mathrm{t}=0 \mathrm{~h}$ and $\mathrm{t}=120 \mathrm{~h}$ ) allowed to investigate the composition of the microbiota. The samples were snap frozen in liquid nitrogen and stored at $\leq-72{ }^{\circ} \mathrm{C}$ until analysis.

\section{Sodium Hydroxide Usage $(\mathrm{pH})$}

The $\mathrm{pH}$ was kept at $\mathrm{pH} 5.8$ by automatic titration with $2 \mathrm{M} \mathrm{NaOH}$.

\section{Short-Chain Fatty Acids and Branched-Chain Fatty Acids}

The dialysate and lumen fractions of TIM-2 were used to analyze SCFA (acetate, propionate and butyrate) and BCFA (iso-butyric acid and iso-valeric acid) with gas chromatography.

For SCFA/BCFA evaluation, samples were prepared and analyzed as described previously [22].

\section{Lactate and Ammonia}

Samples for lactate and ammonia analysis were centrifuged as described above. In the clear supernatant, both 1- and d-lactate were determined enzymatically (based on Boehringer, UV-method, Cat. No. 1112821035, Roche Diagnostics, West Sussex, UK). Ammonia was determined based on the Berthelot reaction [23] in which ammonia reacts first with alkaline phenol and then with sodium hypochlorite to form indophenol blue. In the currently used method, due to its toxicity, phenol was replaced with salicylic acid.

\section{S rDNA Amplicon Sequencing}

The bacterial population in the TIM-2 samples was analyzed using Next Generation sequencing. Total DNA from the collected TIM-2 lumen samples at the start $(\mathrm{t}=0 \mathrm{~h})$ and at the end $(\mathrm{t}=120 \mathrm{~h})$ of the experiments was isolated as described [24] with some minor adjustments: The samples were initially mixed with $250 \mu \mathrm{L}$ lysis buffer (Agowa, Berlin, Germany), $250 \mu \mathrm{L}$ zirconium beads $(0.1 \mathrm{~mm})$, and 
$200 \mu \mathrm{L}$ phenol, before being introduced to a Bead Beater (BioSpec Products, Bartlesville, OK, USA) for 2 min twice. To determine the recovery of bacterial DNA from the samples, a quantitative polymerase chain reaction ( $\mathrm{qPCR}$ ) was used applying universal primers 16Suni-I-F, 5'-CGAAAGCGTGGGGAGCAAA-3' and 16Suni-I-R, 5'-GTTCGTACTCCCCAGGCGG-3', and probe 16Suni-I probe, FAM-5'-ATTAGATACCCTGGTAGTCCA-3'MGB specific for the bacterial 16S rRNA gene. Changes in the microbiota composition were analyzed by using mass V4 16S rDNA amplicon sequencing. For $16 \mathrm{~S}$ rDNA amplicon sequencing of the V4 hypervariable region, $100 \mathrm{pg}$ of DNA was amplified as described [25] using 30 amplification cycles, applying F533/R806 primers [26]. Primers included Illumina adapters and a unique 8-nt sample index sequence key [25]. Amplicon yield, integrity and size was analyzed on a Fragment Analyzer (Advanced Analytical Technologies, Inc., Heidelberg, Germany). The amplicon libraries were pooled in equimolar amounts and purified using agarose gel electrophoresis and subsequent the QIAquick Gel Extraction Kit (QIAGEN, Hilden, Germany). Paired-end sequencing of amplicons was conducted on the Illumina MiSeq platform (Illumina, Eindhoven, The Netherlands).

Processing of the sequencing data was performed using the Mothur pipeline. The differences between the two bacterial community profiles were identified by applying the LEfSe (Linear Discriminant Analysis Effect Size) analysis [27]. The method is based on categorical non-parametric hypothesis test and Linear Discriminant Analysis (LDA) which is a mathematical technique to characterize the difference between classes. This is a method for metagenomic biomarker discovery and therefore allows to find organisms that can help to identify significant differences between two microbial communities. For this a cut-off level of relative abundance of individual genera was included with $0.01 \%$ of total sequences. In the analysis, the different test items were each (as replicate) compared to the control experiments. This shows which genus became significantly more or less abundant as a consequence of a test product compared to the control.

\section{Statistical Analysis}

Mean values of the experiments were compared to mean values of the control experiments.

\section{Results}

\section{Sodium Hydroxide Usage}

During fermentation of carbohydrates the microbiota produces acidic metabolites like SCFA and lactate. The increased use of $\mathrm{NaOH}$ during the experiments for maintenance of $\mathrm{pH}$ at 5.8 indicates the activity of microbiota fermenting the SIEM plus the test product added to the TIM-2 system. Adding Lactulose in the test period $(\mathrm{t}=0 \mathrm{~h}$ to $\mathrm{t}=120 \mathrm{~h})$ showed an increased use of $\mathrm{NaOH}$ during the TIM-2 experiments as compared to the control (Figure 1). Macrogol and Bisacodyl showed a similar total $\mathrm{NaOH}$ usage as in the control experiments at $116 \pm 6 \mathrm{ml}$ (control), $104 \pm 7 \mathrm{ml}$ (Macrogol) and $124 \pm$ $3 \mathrm{ml}$ (Bisacodyl) compared to $436 \pm 2 \mathrm{ml}$ (Lactulose).

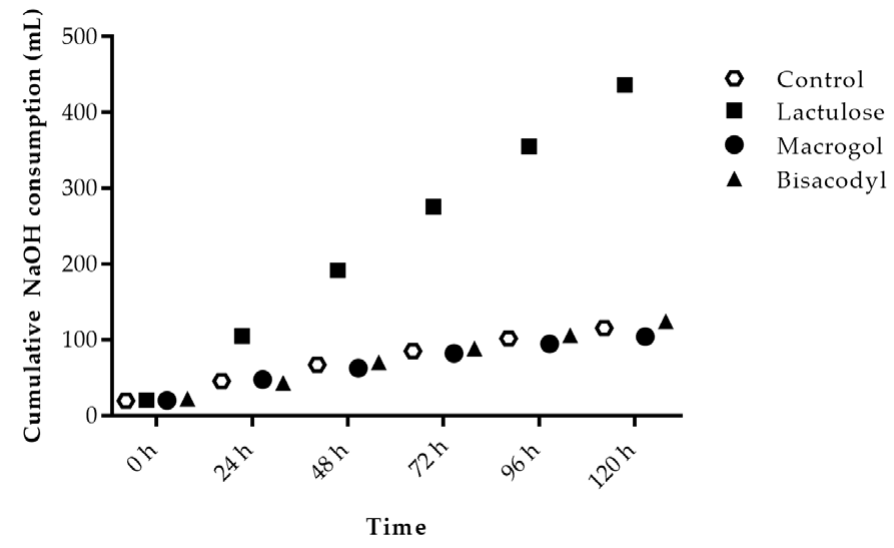

Figure 1: Sodium hydroxide consumption during TIM-2 runs (mean of $\mathrm{n}=3$ (Lactulose), $\mathrm{n}=2$ (Macrogol and Bisacodyl) or $\mathrm{n}=4$ (control)). Values at the start of the test period are on average $20.76 \mathrm{~mL}$ due to $\mathrm{NaOH}$ consumption during the adaptation period. All data points shown at the proximity of the individual time points indicated at the $\mathrm{X}$-axis belong to these specific time points.

\section{Short-chain Fatty Acids and Branched-chain Fatty Acids}

Figure 2a shows the cumulative total SCFA (acetate, propionate and butyrate) production during the $120 \mathrm{~h}$ test period in TIM2. The results indicate that the amount of total SCFA increased with Lactulose $(560 \pm 20 \mathrm{mmol})$, while obtained values for control, Macrogol and Bisacodyl were comparable, with total SCFA amounts of $332 \pm 34 \mathrm{mmol}$ (control), $323 \pm 22 \mathrm{mmol}$ (Macrogol), $351 \pm 17$ mmol (Bisacodyl), respectively.

The total production of branched-chain fatty acids in $120 \mathrm{~h}$ (BCFA; iso-butyrate and iso-valerate) is shown in Figure 2b. During fermentation of proteins in the colon BCFA are produced next to $\mathrm{H}_{2}$, $\mathrm{CO}_{2}, \mathrm{CH}_{4}$, phenols and amines. The total amount of BCFA produced during the TIM-2 experiment was similar for Macrogol $(6.7 \pm 2.7$ $\mathrm{mmol})$ and Bisacodyl $(9.0 \pm 3.1 \mathrm{mmol})$ as compared to the control (8.4 $\pm 4.2 \mathrm{mmol})$, but was lower after addition of Lactulose $(1.2 \pm 0.2 \mathrm{mmol})$.

\section{Lactate}

Lactate is an intermediate metabolite accumulating during fast fermentation processes. At the same time, bacteria use lactate as a substrate. The cumulative amount of lactate (Figure 3) produced in the experiment with Macrogol $(1.2 \pm 0.8 \mathrm{mmol})$ and Bisacodyl $(4.3 \pm$ $2.8 \mathrm{mmol})$ was low and similar to the control $(5.8 \pm 2.2 \mathrm{mmol})$. The results show that due to its fermentation much higher amounts of lactate $(300.7 \pm 10.4 \mathrm{mmol})$ are formed in the presence of Lactulose compared to the control as well as Macrogol and Bisacodyl.

\section{Ammonia}

Ammonia is a metabolite produced by microbial fermentation of proteins (nitrogen). The cumulative (total) amount of ammonia, measured as ammonium salt in the TIM-2 model, is shown in Figure 4. The basal amounts of ammonia (total cumulative production) during the control experiments gives an indication of the ammonia production without intervention. Ammonia production for the different test products was lowest for Lactulose $(22.2 \pm 2.5 \mathrm{mmol})$ compared to $87.0 \pm 27.9 \mathrm{mmol}$ (control), $65.6 \pm 11.2 \mathrm{mmol}$ (Macrogol), and $108.9 \pm 16.8 \mathrm{mmol}$ (Bisacodyl), respectively. 

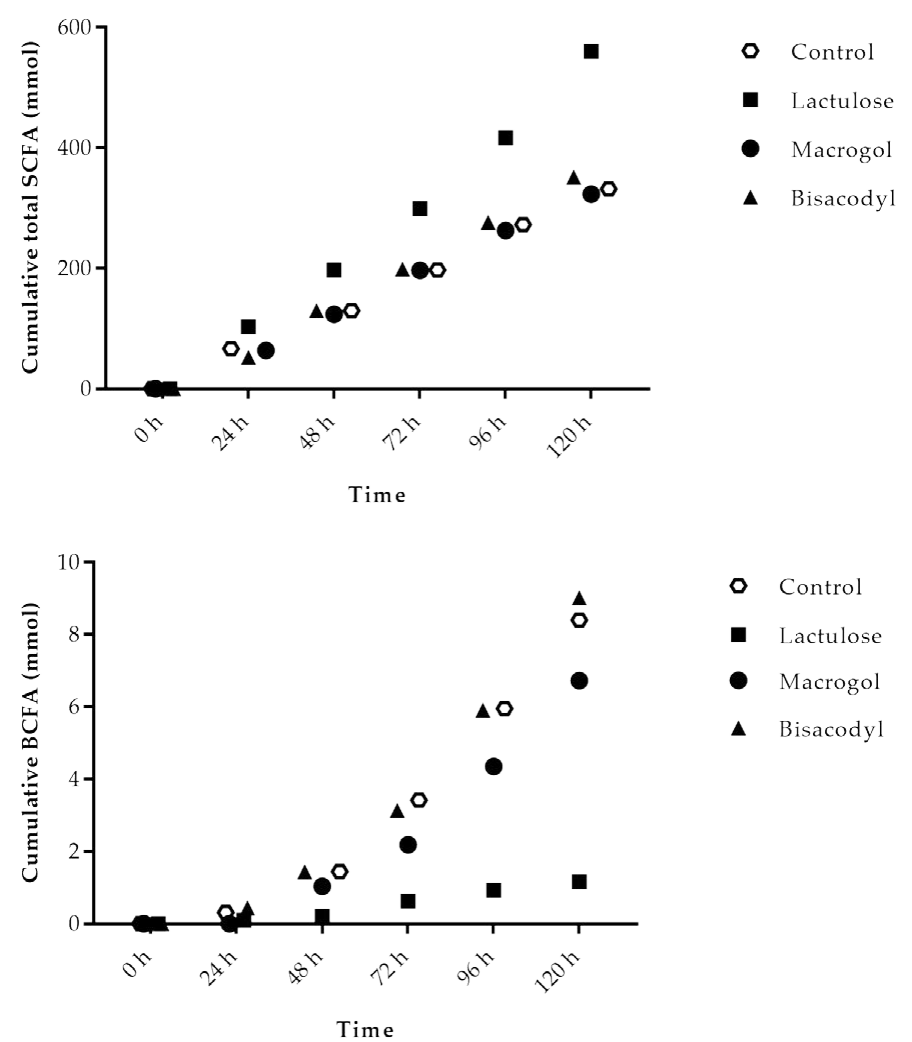

- Control

- Lactulose

- Macrogol

\ Bisacodyl

Figure 2: Production of (A) total short chain fatty acids (SCFA, acetate, propionate, butyrate); (B) total branched-chain fatty acids (BCFA) (iso-butyrate and iso-valerate) in TIM-2 runs (mean of $\mathrm{n}=3$ (Lactulose), $\mathrm{n}=2$ (Macrogol and Bisacodyl) or $\mathrm{n}=4$ (control)). Values at the start of the test period were set to zero. All data points shown at the proximity of the individual time points indicated at the $\mathrm{X}$-axis belong to these specific time points.

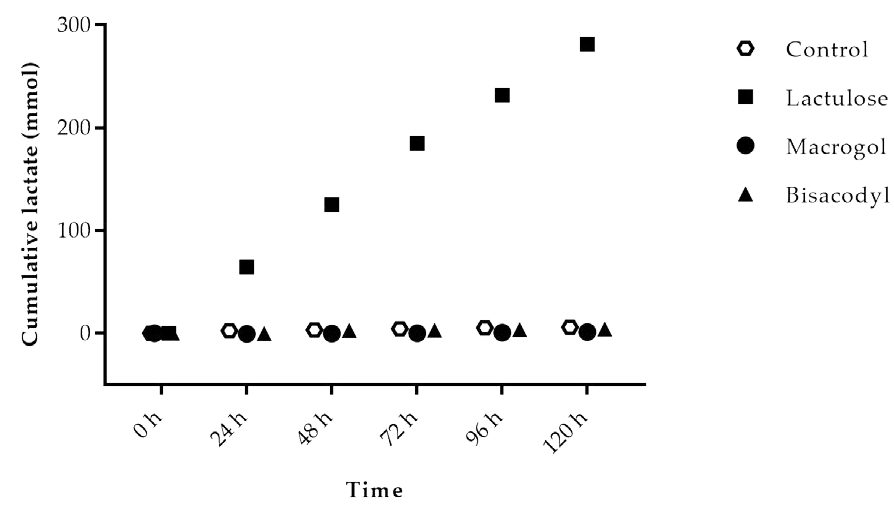

Figure 3: Cumulative lactate production over time during the $120 \mathrm{~h}$ test period in TIM-2 runs (mean of $n=3$ (Lactulose), $n=2$ (Macrogol and Bisacodyl) or $n=4$ (control)). All data points shown at the proximity of the individual time points at the $\mathrm{X}$-axis belong to these specific time points.

\section{Microbiota Composition}

Analysis with mass V4 16S rDNA amplicon sequencing resulted in an overview of bacterial genera present in the microbiota of the lumen samples collected from the TIM-2 experiments after $120 \mathrm{~h}$ exposure to the different test conditions. The distribution of the number of reads ranged from 24,750 to 204,719 . The lowest count of reads observed was 24,750 reads in a $t=0$ sample supplemented with Lactulose. The lowest number of reads was used for normalization of all samples

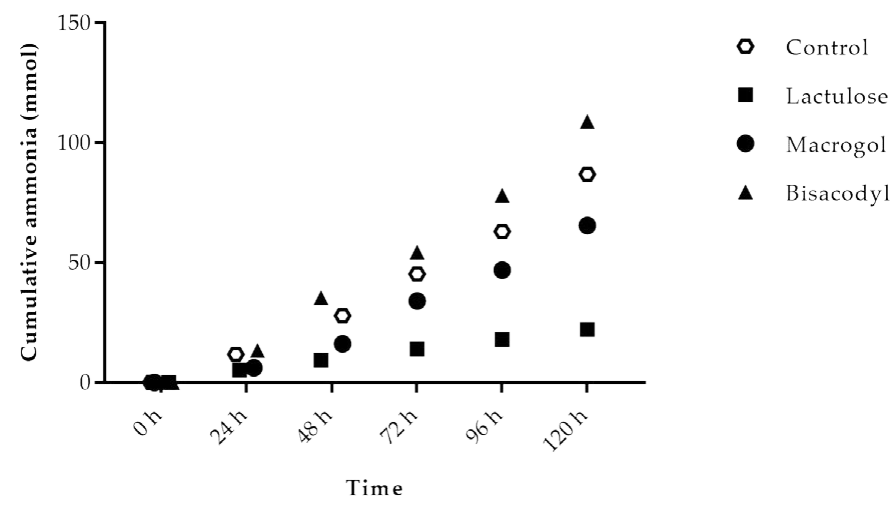

Figure 4: Cumulative ammonia production over time during the $120 \mathrm{~h}$ test period in TIM2 runs (mean of $n=3$ (Lactulose), $n=2$ (Macrogol and Bisacodyl) or $n=4$ (control)). All data points shown at the proximity of the individual time points indicated at the $\mathrm{X}$-axis belong to these specific time points.

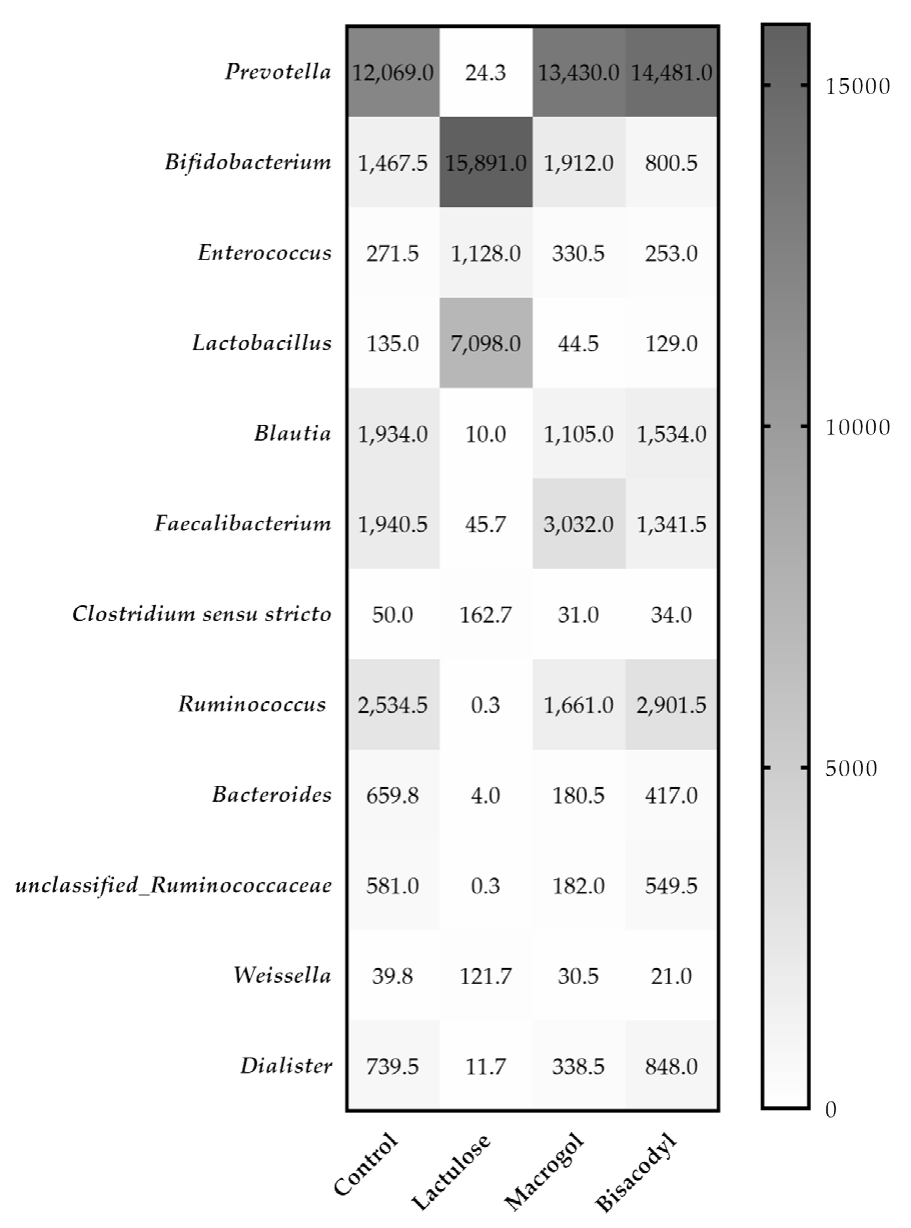

Figure 5: The heatmap indicates the normalized average relative number $\mathrm{n}$ of the different bacterial genera in the microbiota in the different treatments with Lactulose, Macrogol, and Bisacodyl when compared to control, after $120 \mathrm{~h}$ of exposure in TIM-2 as represented by the $16 \mathrm{~S}$ rRNA amplicon sequencing reads.

to this read level. Figure 5 shows the effect of Lactulose, Macrogol and Bisacodyl on the relative abundance of bacteria up to a cut-off range of $1.0 \%$ relative abundance compared to control. The heatmap depicts the most abundant bacteria. The most significantly increased bacterial genera, Bifidobacterium and Lactobacillus increased more than 10 -fold or 50-fold, respectively, in the presence of Lactulose. At 
the same time, Prevotella, Blautia, Ruminococcus, Faecalibacterium and Bacteroides were decreased more than 10 -fold in the presence of Lactulose compared to the control. For the Macrogol and Bisacodyl no significant changes were observed compared to the control.

\section{Discussion}

This study showed that Lactulose, in contrast to Macrogol or Bisacodyl, has an effect on the active gut microbiota present in an in vitro model of the proximal colon. This effect includes an increase in $\mathrm{NaOH}$ consumption to keep the $\mathrm{pH}$ at a fixed level, suggesting a $\mathrm{pH}$ decrease by the net production of acidic metabolic products. This was confirmed by the observed increased levels of SCFA and lactate, and decreased levels in BCFA and ammonia. Contrary to this observation, Bisacodyl even lead to higher cumulative BCFA and ammonia levels than the control.

Five days of exposure to Lactulose strongly increased the levels of bifidobacteria (more than 10-fold) and lactobacilli (more than 50fold). A slight increase in Bifidobacterium was also observed with Macrogol treatment, while Bisacodyl exposure slightly decreased the amount of this bacterium. After Macrogol treatment, the levels of Lactobacillus were decreased, while Bisacodyl exposure had no substantial effect. In summary, exposure to Lactulose was superior to Macrogol and Bisacodyl by increasing the relative abundance of Bifidobacterium and Lactobacillus.

Apart from Bifidobacteria and Lactobacilli, bacterial counts of several other bacteria present in more than $1 \%$ relative abundance with a more than 10-fold changed were observed after Lactulose treatment. Prevotella spp. are reduced close to zero after $120 \mathrm{~h}$ Lactulose treatment, while Macrogol and Bisacodyl treatment slightly increased the counts. Prevotella is suspected to exacerbate chronic (intestinal) inflammation $[28,29]$ and to increase the risk of autoimmune disorders like rheumatoid arthritis [30-34]. Intestinal Prevotellaceae were associated with rheumatoid arthritis in Northern America, Europe and Japan, but not in a Chinese study [35]. Larger metagenome-wide association studies are required before a final conclusion on the role of Prevotella $s p p$ in the pathogenesis of rheumatoid arthritis and the potential for amelioration by Lactulose can be drawn.

Blautia were also reduced nearly 200 -fold by Lactulose, while with both, Macrogol and Bisacodyl, only slight reductions could be identified. An increase in Blautia counts is considered pro-inflammatory [36] and increased counts are detected in neurodegenerative diseases like Parkinson or Multiple Sclerosis [36,37] or systemic lupus erythematosus [38]. The role of increased Blautia counts in the pathogenesis of diabetes is also discussed, but a causative association has not yet been determined [39-41].

Lactulose treatment for $120 \mathrm{~h}$ also showed a decrease in Faecalibacterium, while these bacteria were increased with Macrogol and slightly decreased with Bisacodyl. Within the genus of faecalibacteria, especially Faecalibacterium prausnitzii has been reported as one of the main butyrate producers in the gut $[42,43]$. Due to its anti-inflammatory properties it reduced the severity of inflammation in several murine models [44,45]. The genus Faecalibacterium was also increased in the intestinal content of obese children [46] and patients with psoriasis [47]. The impact of laxatives on levels of this genus remain to be studied in the future.

Ruminococcus was also strongly decreased by Lactulose treatment, slightly decreased by Macrogol and slightly increased by Bisacodyl. While increased levels of these mucolytic bacteria in inflammatory bowel disease (IBD) seem to be associated with the high load of mucins to be cleaved [48,49], nothing is known about the effect of an increased abundance in disorders like autism [50], allergic diseases [48] or coronary artery disease [51].

Finally, Lactulose treatment reduced the levels of Bacteroides, as to a lower extent also did Macrogol and Bisacodyl. This is in contrast to a previous study, where levels of Bacteroides were increased in healthy adults after PEG 4000 induced osmotic diarrhea [11]. The reasons for this difference may be in the test item (PEG 40000 versus PEG 3350), the setup of the study, the dose and the duration of treatment and cannot be fully elucidated here. Bacteroides are normal commensals in the gut, but may also be responsible for infections of significant morbidity, mainly caused by Bacteroides fragilis, like appendicitis, intra-abdominal sepsis, endocarditis, and others [52].

A limitation of this study is the fact that treatment duration was $120 \mathrm{~h}$, while in the clinical situation longer treatment duration may be applicable. A more extended experiment in TIM-2 is possible. Based on previous experience, however, treatment longer than 72-120 h may not reveal significantly different results. Although experiments were conducted only as $\mathrm{n}=2$, results allowed an adequate discrimination between the observed prebiotic effect of Lactulose versus Macrogol and Bisacodyl and more replicates would not have changed this finding.

This study clearly demonstrated that Lactulose has a strong prebiotic effect on Bifidobacteria and Lactobacilli indicating a beneficial support for the gut microbiota of constipated patients in contrast to Macrogol and Bisacodyl. In addition, a more pronounced impact on other gut bacteria was observed with Lactulose compared to Macrogol and Bisacodyl. There are, however, new generations of laxatives also exerting beneficial effects on the gut microbiota or prebiotics with a laxative effect that remain to be compared to Lactulose [53-58]. For example combination products, such as Bisacodyl combined with probiotics [59], or Macrogol mixed with inulin, may result in fierce competition to Lactulose [60]. However, this will remain to be elucidated in future studies, while this study focused on the comparison of the most frequently used single substance laxatives.

\section{Acknowledgments}

We thank Mark Jelier and Eveline Lommen for their excellent technical assistance.

\section{References}

1. Khalif IL, Quigley EM, Konovitch EA, Maximova ID (2005) Alterations in the colonic flora and intestinal permeability and evidence of immune activation in chronic constipation. Dig Liver Dis 37: 838-849. [crossref]

2. Ohkusa T, Koido S, Nishikawa Y, Sato N (2019) Gut Microbiota and Chronic Constipation: A Review and Update. Front Med (Lausanne) 6: 19. [crossref] 
3. Deutsche Gesellschaft für Neurogastroenterologie und Motilität (DGNM) und die Deutsche Gesellschaft für Verdauungs- und Stoffwechselkrankheiten (DGVS) (2013) "Chronische Obstipation: Definition, Pathophysiologie, Diagnostik und Therapie". AWMF-Registriernummer 021/019.

4. Eberlin M, Landes S, Biber-Feiter D, Michel MC (2020) Impact of guideline awareness in public pharmacies on counseling of patients with acute or chronic constipation in a survey of pharmacy personnel. BMC Gastroenterol 20: 191.

5. Gharehbaghi K, Gharehbaghi DR, Wierrani F, Sliutz G (2016) [Treatment of Chronic Functional Constipation during Pregnancy and Lactation]. Z Geburtshilfe Neonatol 220: 9-15. [crossref]

6. Shafe AC, Lee S, Dalrymple JS, Whorwell PJ (2011) The LUCK study: Laxative Usage in patients with GP-diagnosed Constipation in the UK, within the general population and in pregnancy. An epidemiological study using the General Practice Research Database (GPRD). Therap Adv Gastroenterol 4: 343-363. [crossref]

7. Panesar PS, Kumari S (2011) Lactulose: production, purification and potential applications. Biotechnol Adv 29: 940-948. [crossref]

8. Sitanggang AB, Drews A, Kraume M (2016) Recent advances on prebiotic lactulose production. World J Microbiol Biotechnol 32: 154. [crossref]

9. Prasad VG, Abraham P (2017) Management of chronic constipation in patients with diabetes mellitus. Indian J Gastroenterol 36: 11-22. [crossref]

10. Ruszkowski J, Witkowski JM (2019) Lactulose: Patient- and dose-dependent prebiotic properties in humans. Anaerobe 59: 100-106. [crossref]

11. Gorkiewicz G, Thallinger GG, Trajanoski S, et al. (2013) Alterations in the colonic microbiota in response to osmotic diarrhea. PLoS One 8: e55817.

12. van der Wulp MY, Derrien M, Stellaard F, et al. (2013) Laxative treatment with polyethylene glycol decreases microbial primary bile salt dehydroxylation and lipid metabolism in the intestine of rats. Am J Physiol Gastrointest Liver Physiol 305: G474. [crossref]

13. Eduardo J Contijoch, Graham J Britton, Chao Yang, Ilaria Mogno, Zhihua Li et al. (2019) Gut microbiota density influences host physiology and is shaped by host and microbial factors. eLife 8: e40553. [crossref]

14. Tropini C, Moss EL, Merrill BD, et al. (2018) Transient Osmotic Perturbation Causes Long-Term Alteration to the Gut Microbiota. Cell 173: 1742-1754. [crossref]

15. Bustos D, Ogawa K, Pons S, Soriano E, Bandi JC, Bustos Fernandez L (1991) Effect of loperamide and bisacodyl on intestinal transit time, fecal weight and short chain fatty acid excretion in the rat. Acta Gastroenterol Latinoam 21: 3-9. [crossref]

16. Bouhnik Y, Neut C, Raskine L, et al. (2004) Prospective, randomized, parallel-group trial to evaluate the effects of lactulose and polyethylene glycol-4000 on colonic flora in chronic idiopathic constipation. Aliment Pharmacol Ther 19: 889-899. [crossref]

17. Minekus M, Smeets-Peeters M, Bernalier A, et al. (1999) A computer-controlled system to simulate conditions of the large intestine with peristaltic mixing, water absorption and absorption of fermentation products. Appl Microbiol Biotechnol 53: 108-114. [crossref]

18. Venema K, Van Nuenen HMC, Smeets-Peeters M, Minekus M, Havenaar R (2000) TNO's in vitro large intestinal model: an excellent screening tool for functional food and pharmaceutical research. Ernährung/Nutrition 24: 558-564.

19. Maathuis A, Hoffman A, Evans A, Sanders L, Venema K (2009) The effect of the undigested fraction of maize products on the activity and composition of the microbiota determined in a dynamic in vitro model of the human proximal large intestine. J Am Coll Nutr 28: 657-666. [crossref]

20. Maathuis AJ, van den Heuvel EG, Schoterman MH, Venema K (2012) Galactooligosaccharides have prebiotic activity in a dynamic in vitro colon model using (13) C-labeling technique. J Nutr 142: 1205-1212. [crossref]

21. Bothe MK, Maathuis AJH, Bellmann S, et al. (2017) Dose-Dependent Prebiotic Effect of Lactulose in a Computer-Controlled In Vitro Model of the Human Large Intestine. Nutrients 9: 767. [crossref]

22. Van Nuenen M.H., Meyer P., Venema K (2003) The effect of various inulins and clostridium difficile on the metabolic activity of the human colonic microbiota in vitro. Microb Ecol Health Dis 15: 137-144.

23. Sims G., Ellsworth T., Mulvaney R (1995) Microscale determination of inorganic nitrogen in water and soil extracts. Commun Soil Sci Plant Anal 26: 303-316.
24. Ladirat SE, Schols HA, Nauta A, et al. (2013) High-throughput analysis of the impact of antibiotics on the human intestinal microbiota composition. J Microbiol Methods 92: 387-397. [crossref]

25. Kozich JJ, Westcott SL, Baxter NT, Highlander SK, Schloss PD (2013) Development of a dual-index sequencing strategy and curation pipeline for analyzing amplicon sequence data on the MiSeq Illumina sequencing platform. Appl Environ Microbiol 79: 5112-5120. [crossref]

26. Caporaso J.G., Lauber C.L., Walters W.A., et al. (2011) Global patterns of $16 \mathrm{~s}$ rrna diversity at a depth of millions of sequences per sample. Proc Natl Acad Sci USA 18: 4516-4522.

27. Segata N, Izard J, Waldron L, et al. (2011) Metagenomic biomarker discovery and explanation. Genome Biol 12:R60. [crossref]

28. Iljazovic A, Roy U, Galvez EJC, et al. (2021) Perturbation of the gut microbiome by Prevotella spp. enhances host susceptibility to mucosal inflammation. Mucosal Immunol 14: 113-124.

29. Larsen JM (2017) The immune response to Prevotella bacteria in chronic inflammatory disease. Immunology 151: 363-374. [crossref]

30. Guerreiro CS, Calado A, Sousa J, Fonseca JE (2018) Diet, Microbiota, and Gut Permeability-The Unknown Triad in Rheumatoid Arthritis. Front Med (Lausanne) 5: 349. [crossref]

31. Lorenzo D, GianVincenzo Z, Carlo Luca R, et al. (2019) Oral-Gut Microbiota and Arthritis: Is There an Evidence-Based Axis? J Clin Med 8. [crossref]

32. Maeda Y, Takeda K (2017) Role of Gut Microbiota in Rheumatoid Arthritis. J Clin Med 6.

33. Maeda Y, Takeda K (2019) Host-microbiota interactions in rheumatoid arthritis. Exp Mol Med 51: 1-6. [crossref]

34. Wells PM, Williams FMK, Matey-Hernandez ML, Menni C, Steves CJ (2019) 'RA and the microbiome: do host genetic factors provide the link? J Autoimmun 99: 104-115.

35. Moller B, Kollert F, Sculean A, Villiger PM (2020) Infectious Triggers in Periodontitis and the Gut in Rheumatoid Arthritis (RA): A Complex Story About Association and Causality. Front Immunol 11: 1108. [crossref]

36. Frahm C, Witte OW (2019) Mikrobiom und neurodegenerative Erkrankungen. Der Gastroenterologe 14: 166-171.

37. Gerhardt S, Mohajeri MH (2018) Changes of Colonic Bacterial Composition in Parkinson's Disease and Other Neurodegenerative Diseases. Nutrients 10: 708. [crossref]

38. Silverman GJ (2017) Abstract 1786: Patients with SLE show over-representation of Blautia genus in microbiome. American College of Rheumatology Annual Meeting. San Diego.

39. Egshatyan L, Kashtanova D, Popenko A, et al. (2016) Gut microbiota and diet in patients with different glucose tolerance. Endocr Connect 5: 1-9. [crossref]

40. Gurung M, Li Z, You H, Richard Rodrigues, Donald B Jump et al. (2020) Role of gut microbiota in type 2 diabetes pathophysiology. EBioMedicine 51: 102590. [crossref]

41. Jamshidi P, Hasanzadeh S, Tahvildari A, et al. (2019) Is there any association between gut microbiota and type 1 diabetes? A systematic review. Gut Pathog 11: 49. [crossref]

42. Barcenilla A, Pryde SE, Martin JC, et al. (2000) Phylogenetic relationships of butyrate-producing bacteria from the human gut. Appl Environ Microbiol 66: 16541661. [crossref]

43. Duncan SH, Hold GL, Harmsen HJM, Stewart CS, Flint HJ (2002) Growth requirements and fermentation products of Fusobacterium prausnitzii, and a proposal to reclassify it as Faecalibacterium prausnitzii gen. nov., comb. nov. Int J Syst Evol Microbiol 52: 2141-2146. [crossref]

44. Lopez-Siles M, Duncan SH, Garcia-Gil LJ, Martinez-Medina M (2017) Faecalibacterium prausnitzii: from microbiology to diagnostics and prognostics. ISME J 11: 841-852. [crossref]

45. Miquel S, Martin R, Rossi O, et al. (2013) Faecalibacterium prausnitzii and human intestinal health. Curr Opin Microbiol 16: 255-261. [crossref]

46. Balamurugan R, George G, Kabeerdoss J, Hepsiba J, Chandragunasekaran AM, Ramakrishna BS (2010) Quantitative differences in intestinal Faecalibacterium prausnitzii in obese Indian children. Br J Nutr 103: 335-338. [crossref] 
Melanie K. Bothe (2021) Lactulose, but not Macrogol or Bisacodyl, Shows a Prebiotic Effect in a Computer-Controlled In Vitro Model of the Human Large Intestine

47. Codoner FM, Ramirez-Bosca A, Climent E, et al. (2018) Gut microbial composition in patients with psoriasis. Sci Rep 8: 3812. [crossref]

48. Chua HH, Chou HC, Tung YL, et al. (2018) Intestinal Dysbiosis Featuring Abundance of Ruminococcus gnavus Associates With Allergic Diseases in Infants. Gastroenterology 154: 154-167. [crossref]

49. Png CW, Linden SK, Gilshenan KS, et al. (2010) Mucolytic bacteria with increased prevalence in IBD mucosa augment in vitro utilization of mucin by other bacteria. Am J Gastroenterol 105: 2420-2428. [crossref]

50. Wang L, Christophersen CT, Sorich MJ, Gerber JP, Angley MT, Conlon MA (2013) Increased abundance of Sutterella spp. and Ruminococcus torques in feces of children with autism spectrum disorder. Mol Autism 4: 42. [crossref]

51. Toya T, Corban MT, Marrietta E, et al. (2020) Coronary artery disease is associated with an altered gut microbiome composition. PLoS One 15: e0227147. [crossref]

52. Wexler HM (2007) Bacteroides: the good, the bad, and the nitty-gritty. Clin Microbiol Rev 20: 593-621. [crossref]

53. Aguirre M, Bussolo de Souza C, Venema K (2016) The Gut Microbiota from Lean and Obese Subjects Contribute Differently to the Fermentation of Arabinogalactan and Inulin. PLoS One 11: e0159236. [crossref]

54. Buddington RK, Kapadia C, Neumer F, Theis S (2017) Oligofructose Provides Laxation for Irregularity Associated with Low Fiber Intake. Nutrients 9: 1372. [crossref]
55. Macfarlane GT, Steed H, Macfarlane S (2008) Bacterial metabolism and healthrelated effects of galacto-oligosaccharides and other prebiotics. J Appl Microbiol 104: 305-344. [crossref]

56. Martinez RC, Cardarelli HR, Borst W, Simone Albrecht, Henk Schols et al. (2013) Effect of galactooligosaccharides and Bifidobacterium animalis Bb-12 on growth of Lactobacillus amylovorus DSM 16698, microbial community structure, and metabolite production in an in vitro colonic model set up with human or pig microbiota. FEMS Microbiol Ecol 84: 110-123. [crossref]

57. Takayama K, Takahara C, Tabuchi N, Okamura N (2019) Daiokanzoto (Da-HuangGan-Cao-Tang) is an effective laxative in gut microbiota associated with constipation. Sci Rep 9: 3833. [crossref]

58. Van den Abbeele P, Venema K, Van de Wiele T, Verstraete W, Possemiers S (2013) Different human gut models reveal the distinct fermentation patterns of Arabinoxylan versus inulin. J Agric Food Chem 61: 9819-9827. [crossref]

59. Choi YI, Lee JJ, Chung JW, Kyoung Oh Kim, Yoon Jae Kim et al. (2020) Efficacy and Patient Tolerability Profiles of Probiotic Solution with Bisacodyl Versus Conventional Cleansing Solution for Bowel Preparation: A Prospective, Randomized, Controlled Trial. J Clin Med 9: 3286. [crossref]

60. Gruenwald J, Busch R, Bentley C (2009) Efficacy and tolerability of Laxatan Granulat in patients with chronic constipation. Clin Exp Gastroenterol 2: 95-100. [crossref]

Citation:

Bothe MK, Maathuis AJH, Bellmann S, Lange K, van der Vossen JMBM, et al. (2021) Lactulose, but not Macrogol or Bisacodyl, Shows a Prebiotic Effect in a ComputerControlled In Vitro Model of the Human Large Intestine. Microbiol Immunol Pathol Volume 2(3): 1-7. 